\title{
Effect of soft tissue manipulation of popliteus muscle on quadriceps muscle activity and torque production in athletes with anterior knee pain
}

\author{
Srinivasa Rao Pachava, Masoom Goel \\ MYAS-GNDU Department of Sports Sciences and Medicine, Guru Nanak Dev University, Amritsar, Punjab, India
}

\begin{abstract}
Study aim: Popliteus is majorly associated with posterior knee pain; however, this muscle can also have an impact on anterior knee pain. The purpose of this study was to determine the effect of soft tissue manipulation of popliteus muscle on non-traumatic anterior knee pain in athletes; as the relationship between popliteus and Quadriceps muscle has not been studied/explored much.

Materials and methods: In this experimental study, 15 subjects presenting with non-traumatic anterior knee pain were selected. Quadriceps muscle activity, torque production and knee pain were assessed. Followed by a single session of popliteus manipulation all the above parameters were re-assessed immediately and at 24 hours after intervention.

Results: Significant reduction in pain $(\mathrm{P}<0.005)$ at 24 hours was observed. There was a significant increase in muscle activity of Vastus Medialis $(\mathrm{P}<0.05)$ and Vastus Lateralis $(\mathrm{P}<0.005)$ immediately after the intervention but not after 24 hours. No significant change was found in the activity of Rectus femoris and Peak torque of Quadriceps immediately after the intervention and at 24 hours.

Conclusion: Soft tissue manipulation decreased knee pain and improved muscle activity. Therefore, incorporating popliteus manipulation can be effective in treatment of anterior knee pain.
\end{abstract}

Keywords: Electromyography - Manual therapy - Muscle strength - Isokinetic dynamometer

\section{Introduction}

Anterior knee pain (AKP) is one of the most common musculoskeletal complaint seen amongst athletes; with traumatic event, overuse injuries (structures are subject to a tensile force or stress greater than their bearable capacity which leads to pain), idiopathic being some of the main causes. It is non-specific, vague pain that is generally activity-related [23]. It is defined as a self-reported insidious onset of unilateral or bilateral anterior pain, at least two weeks in duration, reproduced with atleast two of the following special tests: patella compression, squatting, prolonged sitting, going up or downstairs, or isometric quadriceps contraction $[31,33]$.

The concept of the amount of load, tissues can tolerate without generating pain has been described very well by Dye et al. [9]. But when a joint or tissue is subjected to increased load or frequency, homeostasis is disrupted, which in turn alters the normal function and henceforth leads to pain [23]. Chronic pain is related to a protective adaptive response in muscle during which the tone of agonist decreases while that of antagonist increases [14, 20]. Greuel et al. [15] studied the isolated effect of pain on torque production and quadriceps activity in anterior knee pain (patellofemoral pain) and observed inhibition in the activity of quadriceps muscle in acute pain. Sterling et al. [44] and Graven-Nielsen et al. [13] suggested that inhibition leads to an increase in the neural drive of voluntary antagonist muscle and muscle imbalance is created. This imbalance and unbalanced forces lead to joint dysfunctions, poor movement patterns, and early fatigue. Poor joint stabilization and stressed muscles are responsible for further injuries. Also, the weakness of the vastus medialis muscle often overloads the Popliteus muscle as it is crucial for terminal extension during the screw home mechanism [30].

Several other studies have also shown that experimental knee pain impairs extensor joint torque (involuntary 
and voluntary quadriceps activation) and rate of force development [32, 37, 12]. Chester et al. [4] observed a delay in recruitment of vastus medialis oblique (VMO) relative to vastus lateralis (VL) muscle in subjects with AKP. Common treatment techniques include patellar taping, soft tissue manipulation, stretching, exercise regimens for the strengthening of vastus medialis muscle correction of abnormal biomechanics [8]. Studies were done to see the "influence of active release technique (ART) on quadriceps inhibition and strength"; and it was concluded that ART protocols did not reduce inhibition or increase strength in the quadriceps muscles of athletes with anterior knee pain [8]. Neither weight-bearing nor non-weight bearing knee-extension exercises acutely increased maximal voluntary quadriceps torque output or activation in patients with AKP [31].

Numerous studies including systemic reviews [22] have been done on myofascial release and the results showed improvements in pain: with fascial manipulation in pubescent postural hyper-kyphosis [5], jaw muscles [16], the range of motion and functional status of individuals suffering from plantar heel pain [1], subacute whiplash disorders [34], carpal tunnel syndrome [36]. However, the effect of myofascial release on anterior knee pain still needs to be studied further.

Popliteal fascia falls in the deep front line while the quadriceps is a part of the superficial front line but they lie in different planes [26]. The deep front line is situated between both lateral lines and is surrounded by spiral lines; it encompasses the myofascial core of the body. Stensdotter et al. [42] studied the popliteus muscle activation in open kinetic chain $(\mathrm{OKC})$ and closed kinetic chain $(\mathrm{CKC})$ knee isometric extension and concluded that popliteus gets activated after quadriceps in knee joint isometric extension and its activation is not anticipatory but rather a part of a prestructured pattern. Based on the tensegrity concept given by Myers [25] in anatomy trains, it is possible to manipulate the soft tissue structure using myofascial release and/or Cyriax techniques. A recent study focusing solemnly on the posterior myofascial train and the effect of myofascial release on pain, range of motion, knee stiffness, and muscle activity, found a significant improvement in mobility and range of motion but it was done on subjects with total knee arthroplasty (TKA) [11].

Current scientific literature lacks discussions about the myofascial association between popliteus and quadriceps muscle. Given there is no direct relationship between the two, no study has been conducted about the relationship between anterior knee pain and popliteus involvement. The previous studies have focused on the rotational aspect during surgical release or solemnly on the posterolateral corner of the knee. As observed in the study [11], myofascial release of the posterior myofascial train had a significant effect on the range of motion and pain, but it was done on total knee arthroplasty subjects. Based on this idea and lack of available literature this study aims at investigating the effect of manipulation of popliteus (one session) on pain, the activity of quadriceps, and torque production of quadriceps. It was hypothesized that manipulation of popliteus muscle will have a significant effect on pain and quadriceps activity.

\section{Materials and methods}

\section{Subjects}

This is an experimental study where 15 subjects (demographic data shown in table 2) with non-traumatic anterior knee pain volunteered to participate after signing informed consent. Inclusion and exclusion criteria are described in table 1 . The data was collected before, immediately after, and at 24 hours post the intervention. This study was in compliance with the Declaration of Helsinki and approved by the Institutional Ethical Committee of Guru Nanak Dev University (40/HG/2020).

Table 1. Inclusion and exclusion criteria

\section{Inclusion criteria:}

- Age group 18 to 25 years.

- Unilateral Subacute or chronic anterior knee pain.

Exclusion criteria:

- Subjects suffering from acute pain.

- History of fracture of knee joint or recent trauma.

- History of knee ligament injury in past six months.

- If currently undergoing any rehabilitative exercise program.

- If currently under any prophylactic medical treatment.

\section{Knee pain and lifestyle outcomek}

The pain was assessed using visual analogue scale (VAS). Lifestyle outcomes were measured using knee injury and osteoarthritis outcome score - patellofemoral subscale (KOOS-PF), [6] to assess the symptoms and functional status of the subject's knee.

\section{Quadriceps torque}

Isokinetic dynamometer (Biodex System 4 Pro; Biodex Medical Systems, Inc, Shirley, NY) was used to assess the isometric strength of the quadriceps muscle for the affected limb at $60^{\circ}$ angle [40]. The validity of Biodex system 3 pro for isometric torque was good enough to be used for both clinical and research purposes [7]. The subjects performed the warmup involving dynamic stretches and slow jog following which they were seated on the isokinetic dynamometer chair with the hip flexed at $90^{\circ}$. Isometric test protocols were set and the knee was positioned at $60^{\circ}$ of flexion. The lower extremity was secured 
Table 2. Demographic data of the subjects

\begin{tabular}{lc}
\hline Parameter $(\mathrm{N}=15)$ & Mean $\pm \mathrm{SD}$ \\
\hline Age & $20.20 \pm 2.40$ \\
Height & $165.25 \pm 8.72$ \\
Weight & $59.80 \pm 10.32$ \\
BMI & $21.87 \pm 2.75$ \\
KOOS score $[\%]$ & $61.73 \pm 14.95$ \\
\hline
\end{tabular}

BMI: Body Mass Index; KOOS score: Knee Injury and Osteoarthritis Outcome Score

to the dynamometer by thigh and ankle straps. Hook and loop straps were used to secure the trunk position and to avoid unnecessary trick movements. 1-2 trial reps were done on healthy limb to make the subject aware about the procedure. Subjects were asked to perform 3 repetitions of 10 seconds each with one-minute rest in between the contractions. They were encouraged by giving visual and verbal feedback. Peak torque of quadriceps was taken for the study. Electromyography (EMG) activity was recorded simultaneously.

\section{Quadriceps muscle activity}

Quadriceps activity was measured by Noraxon telemetric electromyography unit (16 channels) (Noraxon USA. Inc., Scottsdale, AZ, USA). Bipolar Ag-AgCl pregelled surface electrodes, measuring $1 \mathrm{~cm}$ in diameter with a center-to-center distance of $2 \mathrm{~cm}$ were used to record the muscle activity. Before the application of the electrodes, the subject's skin was shaved with a disposable razor and abraded with an alcohol swab. This was done to reduce the skin impedance levels to a level $<5$ $\mathrm{K} \Omega$ before the data collection. For vastus lateralis and vastus medialis oblique, the electrodes were placed on the muscle bellies approximately $8 \mathrm{~cm}$ proximal to the lateral joint line of the knee and approximately $4 \mathrm{~cm}$ proximal to the superomedial border of the patella respectively. The electrodes were placed at the midpoint between the anterior superior iliac spine [ASIS] and the superior pole of the patella for rectus femoris muscle $[21,43]$. The placement points were marked using a marker and the subjects were instructed to keep the marks intact until the next measurement. Adhesive tape was used to secure the transmitter on the skin to avoid any movement artifact. After the electrode placement, subjects were asked to warm up with a brisk walk or slow jog for 1 minute. They were then seated on the Biodex chair; EMG data was recorded simultaneously with the torque production. The testing comprised of 3 repetitions of 10 seconds each with one-minute rest in between the contractions and EMG data was recorded throughout the contraction phase. The contraction phase; lasting 10 seconds was taken for analysis (maximal amplitude values). Later the EMG signals were pre-amplified (gain 400) before transmitting the data in real-time to a 16channel, 16-bit PC-interface receiver (Noraxon Telemyo DTS Telemetry; Noraxon, Arizona, USA). This data was sent via bluetooth and the recorded data was analyzed by Myomuscle V 3.8 clinical applications software. The sampling rate of $1500 \mathrm{~Hz}$ was set within a bandwidth of 10-500 Hz. The signals were rectified and band-pass filtered and smoothened (1000 ms) before being recorded digitally at 1500 samples per second, and then the root mean square (RMS) value was calculated.

\section{Intervention}

After obtaining the pre-data, subjects were asked to lie down on the plinth in prone position with the knee flexed at $30^{\circ}$ to relax the surrounding musculature (Figure 1). First, popliteus was marked to know its exact anatomical location [24]. The lateral femoral condyle (1) and the fibular head (3) were then palpated.

The popliteus tendon passes from the lateral epicondyle to the popliteus muscle at a $45^{\circ}$ angle above the fibular head (3). It emerges from beneath the biceps femoris tendon (line $\mathrm{C}$ ) at approximately the length of the distal phalanx of the thumb from the fibular head and passes diagonally to the muscle belly (line B) [24].
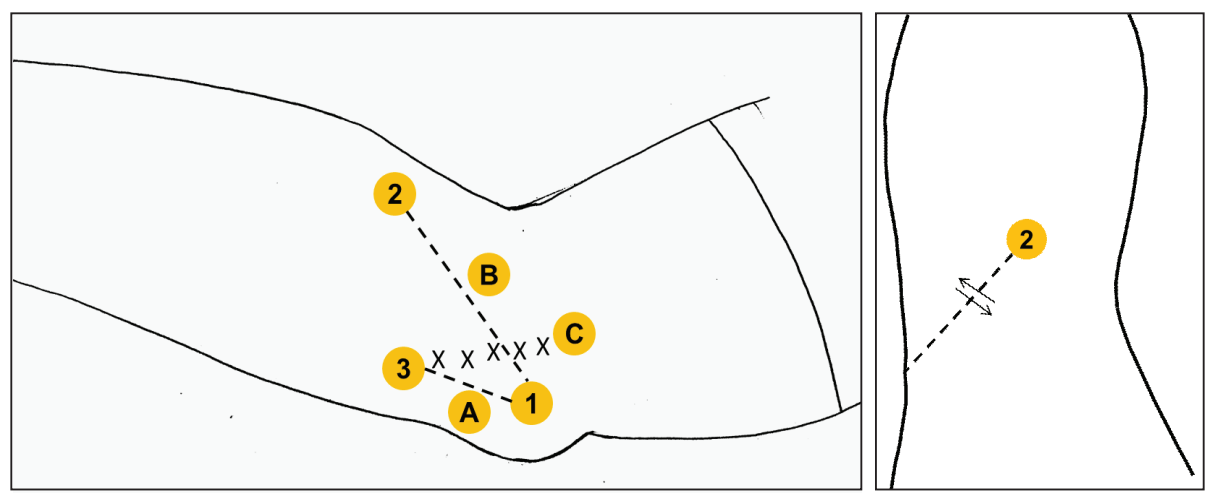

Figure 1. a) Anatomical landmarks (left) and b) Cross friction massage (right) 
Deep tissue friction massage technique [3]; was incorporated with myofascial release however, the duration was decreased to prevent counter-irritation effects and soreness that could have aggravated the pain. Cross friction massage was used due to its deep anatomical location. Strokes were applied across the popliteus tendon, in a path perpendicular to the tendon's orientation, along the entire length of the popliteus tendon and muscle. Three sets of interventions of one-minute duration were given with a rest of 30 seconds in between the sets. Immediately after the procedure; subjects were reseated and data was taken for the experimental limb (no intervention was given thereafter). Data was recorded again after $24 \mathrm{hrs}$.

\section{Statistical analysis}

The SPSS 17 (SPSS Inc., USA) was used for statistical analysis. Tabled data underwent an adherence or normality test (Kolmogorov - Smirnov) that identified all the variables as normally distributed. Whole data was described in terms of mean and standard deviation. Paired t-test was used to compare mean values of RMS, peak torque, and knee pain before and immediately after intervention and at $24 \mathrm{hrs}$. Repeated ANOVA was used to see the difference within the group. Significance level was defined as $95 \%$ confidence interval with $p$-value $\leq 0.05$.

\section{Results}

The mean and standard deviations of all the recorded variables are reported in table 3 and 4.

Table3. Difference in VAS, before, and 24 hours after intervention

$\begin{array}{lcc} & \text { Pre } & 24 \text { hours Post } \\ \text { Parameter } & \text { Mean } \pm \text { SD } & \text { Mean } \pm \text { SD } \\ \text { VAS } & 6.33 \pm 1.496 & 4.47 \pm 1.552 * *\end{array}$

** Significant for P-value $<0.005$; VAS: Visual Analogue Scale.

\section{Knee pain}

A significant difference $(\mathrm{P}<0.005)$ was observed in the VAS score before the intervention and 24 hours after intervention (table 3).

\section{Muscle activity and torque production}

When the EMG activity of the RF, VMO, and VL before intervention was compared with that of immediately after intervention significant increase in the activity of VMO $(\mathrm{P}<0.05)$ and VL $(\mathrm{P}<0.005)$ was observed. There was no significant difference observed in any of the muscles when the activity before intervention was compared with activity 24 hours after the intervention (table 4 ).

The peak torque of quadriceps muscle, immediately after and 24 hours after intervention showed no significant difference from that of before intervention (table 4).

When the data of muscle activity and peak torque was checked for variance (shown as F-value in table 4), no significant differences were found within the group.

\section{Discussion}

This study aimed to investigate the acute effects of popliteus muscle manipulation on quadriceps muscle activity, torque production, and knee pain. The results indicated a significant improvement in pain at $24 \mathrm{hrs}$ and activity of vastus medialis oblique and vastus lateralis immediately after the intervention. No significant improvement was observed in rectus femoris activity and torque production.

In anterior knee pain, VMO and VL are more commonly inhibited with an alteration in myoelectric signals resulting in pain $[4,29,35,46,47]$. But these studies focused on the onset timing of VMO and VL firing rather than amplitude and found delayed VMO recruitment. In painful knees, there is a compensatory increase in muscle activation by increasing tensile forces produced by muscles acting to pull the tibia and femur together in order to increase contact mechanics and joint stiffness [13]. Secondary complications associated with anterior knee pain include changes in soft tissue and muscle tone such as weakness of VMO

Table 4. Differences in EMG activity and peak torque before, immediately after, and 24 hours after intervention

\begin{tabular}{lcccc}
\hline \multirow{2}{*}{ Parameters } & Pre & Immediate post & 24 Hours post & Mean \pm SD \\
\cline { 2 - 4 } & Mean \pm SD & Mean \pm SD & Malue & 1.02 \\
RF $(\mathrm{mV})$ & $0.48 \pm 0.214$ & $0.51 \pm 0.236$ & $0.47 \pm 0.177$ & 1.80 \\
VMO $(\mathrm{mV})$ & $0.70 \pm 0.358$ & $0.80 \pm 0.364^{*}$ & $0.80 \pm 0.285$ & 1.70 \\
VL $(\mathrm{mV})$ & $0.62 \pm 0.382$ & $0.71 \pm 0.360^{* *}$ & $0.63 \pm 0.405$ & 1.94 \\
Quadriceps Torque (FT-LBS) & $102.41 \pm 43.800$ & $108.01 \pm 48.202$ & $115.19 \pm 37.943$ & 1.94 \\
\hline
\end{tabular}

* Significant at $\mathrm{p}<0.05$; * Significant at $\mathrm{p}<0.005$; RF: Rectus Femoris; VMO: Vastus Medialis Oblique; VL: Vastus Lateralis; mV: milli volts; FT-LBS: foot-pounds. 
and spasm of the popliteus muscle. Weakness of the vastus medialis muscle often overloads the popliteus muscle as it is crucial for terminal extension during screw home mechanism [30]. Experimental knee pain using hypertonic saline injections has also shown a significant reduction in vastus medialis amplitude and knee extensor torque [14]. Primarily, popliteus acts as a dynamic restraint to tibial external rotation between $20^{\circ}$ to $30^{\circ}$ of knee flexion [28]. Studies have shown that although popliteus muscle gets activated after the quadriceps in isometric knee extension especially in the open kinetic chain, at $30^{\circ}$ of knee flexion its peak amplitude is greater than quadriceps muscle [42].

Massage causes a reduction in motor neuron excitability due to decrease in H-reflex and hence creates an inhibitory effect on the muscle being focused in neurologically sound subjects [45]. Significant reduction in pain and increase in activity of VMO and VL after soft tissue manipulation i.e. deep tissue friction massage which led to tight popliteus inhibition is in accordance with this. Stensdotter et al. [43] concluded that in OKC amplitude of VMO is less than VL. In the present study, activity of VL and VMO increased significantly immediately after the intervention with VL showing much significant improvement than VMO. It may be due to the fact that popliteus is primarily a part of the posterolateral complex [28] and its manipulation might affect Vastus Lateralis activity directly. But this increase didn't persist at $24 \mathrm{hrs}$ duration which is in contrast to the study by Henriksen et al, [19] where they concluded that knee joint pain and muscle strength inhibition are strongly and positively correlated. However, the presence of sustained muscle inhibition even after pain relief is not uncommon [10,17,18,39,41]. Studies have also shown that either stronger forces or longer durations are required for permanent viscoelastic deformation of fascia [38].

Previously it has been observed that pain impairs joint force and torque development [12, 32, 37]. In this study though favorable the increase in torque production was not significant inspite of pain relief at $24 \mathrm{hrs}$. Since the subjects were not rested post-intervention i.e. they were allowed to continue with their routine training which could have affected the muscle activity as well as torque production. The effect of co-activation of other antagonists i.e. hamstrings and gastrocnemius on net extensor torque [2] was also not considered.

E Silva et al. [11] conducted a study on subjects who underwent total knee arthroplasty surgery and found a significant effect on pain, range of motion, and biceps femoris activity in subjects with flexion contracture post-surgery, immediately after the release of posterior myofascial chain. This study, focused just on popliteus muscle which although a part of the posterolateral complex; belongs to the deep front line [27], and found a similar improvement in pain but not in rectus femoris activity. A single session of popliteus manipulation might be effective in improving knee pain and muscle activity but not the torque production of quadriceps muscle.

\section{Limitations}

Due to COVID 19 pandemic, the data collection process was affected and was limited to a smaller sample. A larger sample is strongly indicative to obtain an absolute conclusion. Also, this study focused on a single session of manipulation, future studies may focus on more sessions of this protocol.

\section{Conclusion}

In subacute and chronic knee pain, incorporating popliteus manipulation along with other treatment techniques may effectively decrease pain, improve muscle activity and help in early return to sports. Along with the pain relief, the possibility of performance enhancement can't be neglected. Rather than just focussing on larger group of muscles equal importance should be given to the smaller and the important ones in the kinetic chain to enhance recovery and prevent re-injuries. A sports-specific study would help to understand the role of popliteus muscle in different types of sports. The role of rectus femoris in anterior knee pain is an area that needs to be explored as this and previous studies are inconclusive.

\section{Conflict of interest: Authors state no conflict of interest.}

\section{References}

1. Ajimsha M.S., Binsu D., Chithra S. (2014) Effectiveness of myofascial release in the management of plantar heel pain: a randomized controlled trial. Foot., 24(2): 66-71. DOI: 10.1016/j.foot.2014.03.005.

2. Alkner B.A., Tesch P.A., Berg H.E. (2000) Quadriceps EMG/force relationship in knee extension and leg press. Med. Sci. Sports Exerc., 32(2): 459-463. DOI: 10.1097/00005768-200002000-00030.

3. Chamberlain G.J. (1982) Cyriax's friction massage: a review. J. Orth. Sport Phys. Ther., 4(1): 16-22. DOI: 10.2519/jospt.1982.4.1.16.

4. Chester R., Smith T.O., Sweeting D., Dixon J., Wood S., Song F. (2008) The relative timing of VMO and VL in the aetiology of anterior knee pain: a systematic review and meta-analysis. BMC Musculoskelet. Disord., 9(1): 1-14. DOI: 10.1186/1471-2474-9-64.

5. Ćosić V., Day J.A., Iogna P., Stecco A. (2014) Fascial Manipulation ${ }^{\circledR}$ method applied to pubescent postural hyperkyphosis: A pilot study. J. Bodyw. Mov. Ther, 18(4): 608-615. DOI: 10.1016/j.jbmt.2013.12.011. 
6. Crossley K.M., Macri E.M., Cowan S.M., Collins N.J., Roos E.M. (2018) The patellofemoral pain and osteoarthritis subscale of the KOOS (KOOS-PF): development and validation using the COSMIN checklist. Br. J. Sports Med., 52(17): 1130-1136. DOI: 10.1136/bjsports-2016096776.

7. Drouin J.M., Valovich-McLeod T.C., Shultz S.J., Gansneder B.M., Perrin D.H. (2004) Reliability and validity of the Biodex system 3 pro isokinetic dynamometer velocity, torque and position measurements. Eur. J. Appl. Physiol., 91(1): 22-29. DOI: 10.1007/s00421-0030933-0.

8. Drover J.M., Forand D.R., Herzog W. (2004) Influence of active release technique on quadriceps inhibition and strength: a pilot study. J. Manipulative Physiol. Ther., 27(6): 408-413. DOI: 10.1016/j.jmpt.2004.05.006.

9. Dye S.F., Stäubli H.U., Biedert R.M., Vaupel G.L. (1999) The mosaic of pathophysiology causing patellofemoral pain: Therapeutic implications. Oper. Tech. Sports Med., 7(2): 46-54. DOI: 10.1016/S1060-1872(99)80014-8.

10. De Silva D.C.C.M., de Andrade Alexandre D.J., Silva J.G. (2018) Immediate effect of myofascial release on range of motion, pain and biceps and rectus femoris muscle activity after total knee replacement. J. Bodyw. Mov. Ther., 22(4): 930-936. DOI: 10.1016/j.jbmt.2017.12.003.

11. Flaxman T.E., Shourijeh M.S., Alkjær T., Krogsgaard M.R., Simonsen E.B., Bigham H., Benoit D.L. (2019) Experimental muscle pain of the vastus medialis reduces knee joint extensor torque and alters quadriceps muscle contributions as revealed through musculoskeletal modeling. Clin. Biomech., 67: 27-33. DOI: 10.1016/j. clinbiomech.2019.04.005.

12. Graven-Nielsen T., Lund H., Arendt-Nielsen L., Danneskiold-Samsøe B., Bliddal H. (2002) Inhibition of maximal voluntary contraction force by experimental muscle pain: a centrally mediated mechanism. Muscle Nerve, 26(5): 708-712. DOI: 10.1002/mus. 10225.

13. Graven-Nielsen T., Svensson P., Arendt-Nielsen L. (1997) Effects of experimental muscle pain on muscle activity and co-ordination during static and dynamic motor function. Electroencephalogr. Clin. Neurophysiol., 105(2): 156-164. DOI: 10.1016/s0924-980x(96)96554-6.

14. Greuel H., Herrington L., Liu A., Jones R.K. (2019) How does acute pain influence biomechanics and quadriceps function in individuals with patellofemoral pain?. Knee, 26(2): 330-338. DOI: 10.1016/j.knee.2018.12.008.

15. Guarda-Nardini L., Stecco A., Stecco C., Masiero S., Manfredini D. (2012) Myofascial pain of the jaw muscles: comparison of short-term effectiveness of botulinum toxin injections and fascial manipulation technique. Cranio., 30(2): 95-102. DOI: 10.1179/crn.2012.014.

16. Lund J.P., Donga R., Widmer C.G., Stohler C.S. (1991) The pain-adaptation model: a discussion of the relationship between chronic musculoskeletal pain and motor activity. Can. J. Physiol. Pharmacol., 69(5): 683-694. DOI: $10.1139 / \mathrm{y} 91-102$.

17. Matheson J.W., Kernozek T.W., Fater D.C., Davies G.J. (2001) Electromyographic activity and applied load during seated quadriceps exercises. Med. Sci. Sports Exerc., 33(10): 1713-1725. DOI: 10.1097/00005768-20011000000016.

18. McKenney K., Elder A.S., Elder C., Hutchins A. (2013) Myofascial release as a treatment for orthopaedic conditions: a systematic review. J. Athl. Train., 48(4): 522-527. DOI: 10.4085/1062-6050-48.3.17.

19. Morgan T., Stevens S.D., Palmer T. (2007) Popliteus dysfunction and manual therapy. Int. J. Athl. Ther. Train., 12(6): 16-19. DOI: 10.1123/att.12.6.16.

20. Myers T.W. (2013) Anatomy trains e-book: myofascial meridians for manual and movement therapists. Elsevier Health Sciences.

21. Myers T.W. (2009) Anatomy Trains: Myofascial meridians for manual and movement therapists, Churchill Livingstone.

22. Myers T.W. (2010) Anatomy Trains: Myofascial meridians for manual and movement therapists, Churchill Livingstone.

23. Nahas S., Patel A., Hodgson H., Gupte C. (2019) The posterolateral corner of the knee. Orthop. Trauma., 33(2): 100-108. DOI: 10.1016/j.mporth.2019.01.003.

24. Owings T.M., Grabiner M.D. (2002) Motor control of the vastus medialis oblique and vastus lateralis muscles is disrupted during eccentric contractions in subjects with patellofemoral pain. Am. J. Sports Med., 30(4): 483-487. DOI: $10.1177 / 03635465020300040601$.

25. Page P., Frank C.C., Lardner R. (2010) Assessment and treatment of muscle imbalance: the Janda approach. Human Kinetics.

26. Park J., Grindstaff T.L., Hart J.M., Hertel J.N., Ingersoll C.D. (2012) Knee-extension exercise's lack of immediate effect on maximal voluntary quadriceps torque and activation in individuals with anterior knee pain. J. Sport Rehabil., 21(2): 119-126. DOI: 10.1123/jsr.21.2.119.

27. Park J., Hopkins J.T. (2013) Induced anterior knee pain immediately reduces involuntary and voluntary quadriceps activation. Clin. J. Sport Med., 23(1): 19 24. DOI: 10.1097/jsm.0b013e3182717b7b.

28. Patel D.R., Villalobos A. (2017) Evaluation and management of knee pain in young athletes: overuse injuries of the knee. Transl. Pediatr., 6(3): 190. DOI: 10.21037/ tp.2017.04.05.

29. Picelli A., Ledro G., Turrina A., Stecco C., Santilli V., Smania N. (2011) Effects of myofascial technique in patients with subacute whiplash associated disorders: a pilot study. Eur. J. Phys. Rehabil. Med., 47(4): 561-568. PMID: 21796089.

30. Portney L.G., Sullivan P.E., Daniell J.L. (1986) EMG activity of vastus medialis obliquus and vastus lateralis in 
normal and patients with patellofemoral arthralgia. Phys. Ther., 66: 808.

31. Pratelli E., Pintucci M., Cultrera P., Baldini E., Stecco A., Petrocelli A., Pasquetti P. (2015) Conservative treatment of carpal tunnel syndrome: Comparison between laser therapy and fascial manipulation ${ }^{\circledR}$. J. Bodyw. Mov. Ther., 19(1): 113-118. DOI: 10.1016/j.jbmt.2014.08.002.

32. Rice D.A., Mannion J., Lewis G.N., McNair P.J., Fort L. (2019) Experimental knee pain impairs joint torque and rate of force development in isometric and isokinetic muscle activation. Eur. J. Appl. Physiol., 119(9): 2065-2073. DOI: 10.1007/s00421-019-04195-6.

33. Schleip R. (2003) Fascial plasticity-a new neurobiological explanation: Part 1. J. Bodyw. Mov. Ther, 7(1): 11-19. DOI: 10.1016/S1360-8592(02)00067-0.

34. Shenoy S., Mishra P., Sandhu J.S. (2011) Peak torque and IEMG activity of quadriceps femoris muscle at three different knee angles in a collegiate population. $J$. Exerc. Sci. Fit., 9(1): 40-45. DOI: 10.1016/S1728-869X(11)60005-1.

35. Stensdotter A.K., Dalén T., Holmgren C., Häger-Ross C. (2008) Knee angle and force vector - dependent variations in open and closed kinetic chain for M. popliteus activation. J. Orthop. Res., 26(2): 217-224. DOI: 10.1002/ jor.20491.

36. Stensdotter A.K., Hodges P., Mellor R., Sundelin G., Häger-Ross C. (2003) Quadriceps activation in closed and in open kinetic chain exercise. Med. Sci. Sports Exerc., 35(12): 2043-2047. DOI: 10.1249/01. mss.0000099107.03704.ae.
37. Sterling M., Jull G., Wright A. (2001) The effect of musculoskeletal pain on motor activity and control. J. Pain, 2(3): 135-145. DOI: 10.1054/jpai.2001.19951.

38. Sullivan S.J., Williams L.R., Seaborne D.E., Morelli M. (1991) Effects of massage on alpha motoneuron excitability. Phys Ther., 71(8): 555-560. DOI: 10.1093/ $\mathrm{ptj} / 71.8 .555$.

39. Van Tiggelen D., Cowan S., Coorevits P., Duvigneaud N., Witvrouw E. (2009) Delayed vastus medialis obliquus to vastus lateralis onset timing contributes to the development of patellofemoral pain in previously healthy men: a prospective study. Am. J. Sports Med., 37(6): 1099-1105. DOI: 10.1177/0363546508331135.

40. Wong Y.M. (2009) Recording the vastii muscle onset timing as a diagnostic parameter for patellofemoral pain syndrome: fact or fad? Phys. Ther. Sport, 10(2): 71-74. DOI: $10.1016 /$ j.ptsp.2009.02.001.

\section{Received 23.09.2021 \\ Accepted 30.11.2021}

(C) University of Physical Education, Warsaw, Poland

Acknowledgments

The authors would like to sincerely thank the participants of the study and MYAS-GNDU Department of Sports Sciences and Medicine for their invaluable support. 